\title{
Human Activity Recognition Using Smartphone and Smartwatch
}

\author{
Hamid M. Ali ${ }^{1}$, Ali M. Muslim² \\ ${ }^{1}$ Computer Engineering Dept., Baghdad University, Iraq, habdul hussain@yahoo.com \\ ${ }^{2}$ Computer Engineering Dept., Baghdad University, Iraq, alimajeed.mail@gmail.com
}

\begin{abstract}
Human activity recognition is influential subject in different fields of human daily life especially in the mobile health. As the smartphone becomes an integrated part of human daily life which has the ability of complex computation, internet connection and also contains a large number of hardware sensors, encourage implementation of the human activity recognition system. Most of the works done in this field imposed the restriction of firmly fixing the smartphone in a certain position on the human body, together with machine learning mechanism, to facilitate the process of classifying human activities from the smartphone sensors raw data. To overcome this restriction, the proposed approach incorporated a smartwatch, fixed on the human ankle, together with smartphone freely carried by the user. The use of smartwatch assisted in providing distinctly separable signal variation from the smartwatch accelerometer and gyroscope sensors raw data which in turn facilitated the use of a threshold-based mechanism to classify 20 various human activities. Furthermore, this work provides a service for remotely real-time monitoring of the user human activities the system is tested with different subjects and achieved an accuracy of $97.5 \%$.
\end{abstract}

Keywords- Real-time monitoring, human activity recognition, threshold-based mechanism, mHealth, smartphone, and smartwatch.

\section{INTRODUCTION}

Recently, the smartphone becomes an integrated part of human daily life and is turned into more and more sophisticated device with growing the computation power, network capability and sensing powers. Also, it contains a large number of hardware sensors such as accelerometer, gyroscope, compasses, barometer, temperature, humidity, light sensor and GPS receiver. All these features encourage implementation of human activity recognition system and make the smartphone to become a rich environment for many systems like healthcare system called Mobile Health (mHealth) system. Mobile Health system is the intersection between Electronic Health (eHealth) and smartphone technology [1]. Smartphone sensors have become a source data to measure various human activities, for example, physical states such as running, walking, walking upstairs and downstairs. Monitoring of these activities can be used to predict user health and provide health care services at the right time. In the last few years, several works have been established using a smartphone as a tool for building human activity recognition system. The authors in [2] used smartphone fixed at the position around waists such as jacket pocket and pants pocket, with arbitrary orientation using a quadratic classifier, k-nearest neighbor algorithm, support vector machine, and artificial neural networks as classifiers to classify 5 activities. Actually the lower number of activities, 5 only, not enough to predict user health. The accuracy achieved by this research is $84.4 \%$. In [3] the authors used fixed smartphone in the front pocket of the user trouser using both Clustered KNN and Naïve Bayes as classifiers to recognize 4 activities. Actually the lower number of activities, 4 only, not enough to predict user health. The best performance achieved by this research is $92 \%$. In [4] the author's used smartphone fixed on the user's chest using SVM as a classifier to recognize 16-activities. The total recognition rate of $95.03 \%$ is achieved by this work. In [5] the author's used smartphone fixed to one of the four positions on the waist, wrist, chest and thighs using SVM as classier to classify six activities.

Actually the lower number of activities, 6 only, not enough to predict user health. The accuracy achieved by this research is $96 \%$. In [6] the authors used smartphone fixed in one of the user trouser pockets in addition to a fixed wearable sensor in the user's chest (Zephyr BioHarness) using Weka machine learning package to recognize 12- activities. The best performance achieved by this research is $95 \%$. The Zephyr BioHarness sensor is 
not easy to use for the patient people because it is relatively big and it hinders breathing by tightening it around the chest. In [7] the authors used a smartphone fixed in one of the front-right and the back-left user trouser pockets using a neural network as a classifier to recognize 5 activities, the overall accuracy is $93.311 \%$. The training data sets for this work is difficult and takes a long time because the training of the neural network needs to be performed by using MATLAB on a desktop workstation. In [8] the author's used smartphone fixed on one of five body positions, right jeans pocket, left jeans pocket, belt position, right upper arm and right wrist using SVM as a classifier to recognize 7-activities. The highest classification accuracy achieved by this research is $82 \%$.

To obtain best results from the smartphone sensors raw data, all the related work imposed the restriction of fixing the smartphone on the human body. Actually, this restriction is cumbersome and prevents the user from using the smartphone for the purpose of calling, serving the internet, social media etc. The rest of the paper is organized as follows: Section 2 describes system approach mechanism, section 3 presents the results and performance achieved from real test, section 4 discusses the obstacles and challenges encountered in human activities recognition, section 5 lists and justifies the smartwatch role in achieving more accurate results, section 6 concludes the paper.

\section{SYSTEM APPROACH}

To the best of the researchers' knowledge that the most up-to-date approaches, conducted in this field, depend on the smartphone inertial sensors to accomplish human activity recognition. The main contribution of this work is the incorporation of the android smartwatch as a part of the system components which in turn is used in conjunction with a smartphone for better accurate human activity recognition results. Figure 1 shows the main system components of the overall system structure.

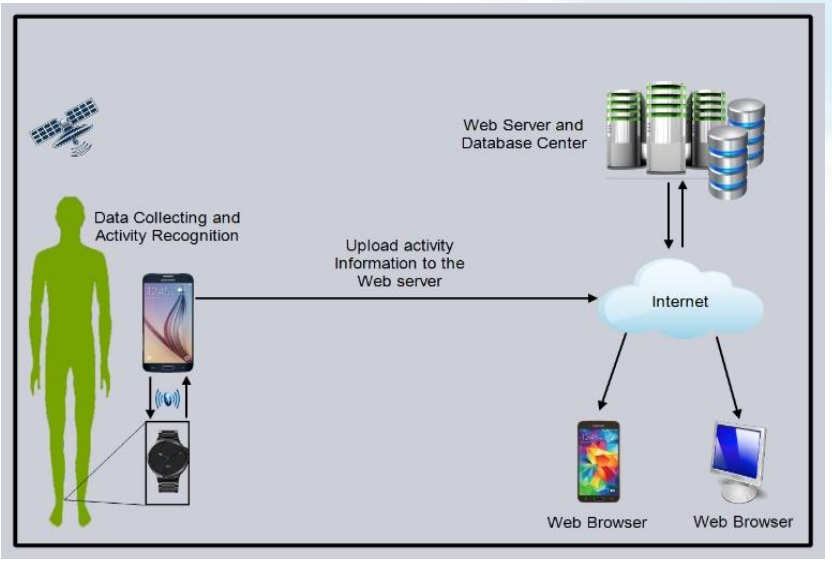

Figure 1 Overall system structure

The main restriction that is imposed by the existing systems is: Fixing firmly the smartphone on the human body; actually this is not a realistic solution, as the user sometimes wants to serve in the smartphone which implies holding the smartphone in different positions and is then subjected to drastic movements. This maneuver affects the smartphone inertial sensors coordinate's readings which could then produce inaccurate activity recognition results. As a consequence, the proposed system integrated the use of smartwatch fixed on user's ankle together with smartphone carried, by the user, in any position and direction.

Figure 2 illustrates the position and direction of placing and fixing the smartwatch on the right human anklefoot. The main technical reason behind using the smartwatch is to place the smartwatch sensors (accelerometer and gyroscope coordinates) in a position that become steady and precisely sensitive to human body movements. In other words, the mentioned smartwatch sensors can provide more generalized and separable features to be exploited for more accurate results in the human activity recognition system.

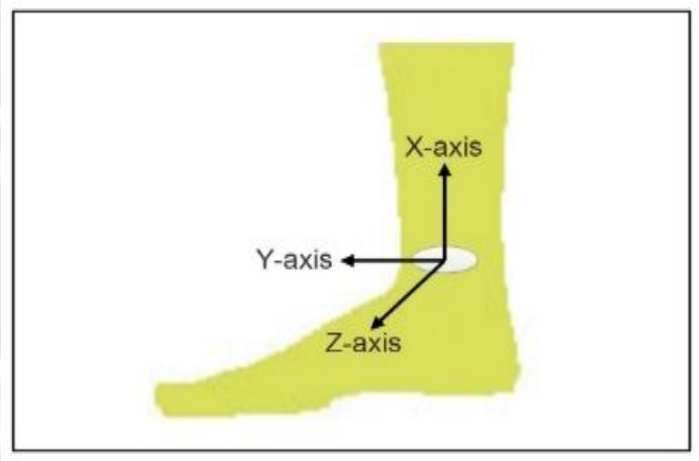

Figure 2: Smartwatch position and orientation

Figure 3 shows the overall system design mechanism and it's interoperated components that are working simultaneously to achieve the intended human activity recognition system. Furthermore, this work provides a service for real-time monitoring of user human activities which is accomplished by continuously sending user identified activities to a database server to be accessed by web browser by authorized people. The system is implemented under Android Platform. 


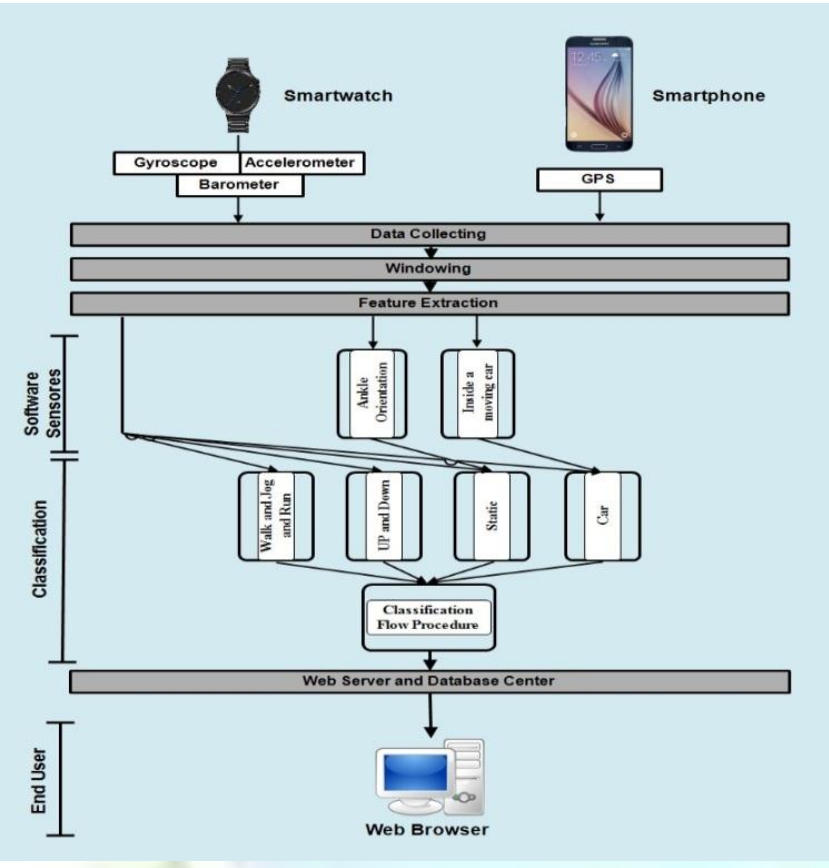

Figure 3: Proposed System Design

\subsection{Data collection}

The data collection component integrated into the smartwatch and the smartphone, in the smartwatch to collect data from smartwatch sensors while in the smartphone to collect GPS data. The smartwatch collects data from their sensors at the rate of every $20 \mathrm{~ms}$, the sensor sampling rates are chosen carefully after conducting many experimental tests with different situations to obtain the best clear features that are suitable for all activities to achieve minimum processing load and separable/generalized features.

\subsection{Windowing}

The windowing process is used to split the continuously collected data, of each sensor, into small time segments called windows for the purpose of feature extraction. In this work, a window size of 3-seconds is adapted to be adequate for feature extraction. The windows size is chosen after having thoroughly analyzed the intended human activities to satisfy reliable activity recognition with high accuracy. For example, increasing the window size could miss detecting short activity like up and down stairs especially in case there is a stair with a halflanding between floors. On the other hand, a short window size increases the misclassification because some features would not be clear to recognize. The windowing process is executed on the smartwatch.

\subsection{Feature Extraction}

Each smartwatch sensors window size exemplifies 150 vectors of raw data; some sensors have 3-axes coordinates. The purpose of feature extraction process is to inspect and measure the properties of the window raw data pattern relative to the required activity recognition. In another word, to find the best approximate distribution of the raw data to which activity it belongs. In time domain, the total features that are analyzed and extracted for this work, concerning each human activity, are shown in table 1. Also, the feature extraction stage is executed in the smartwatch.

Table 1: Total extracted feature values for a window

\begin{tabular}{|l|l|}
\hline No & Feature \\
\hline 1 & Mean of the smartwatch accelerometer $x$-axis \\
\hline 2 & Mean of the smartwatch accelerometer y-axis \\
\hline 3 & Mean of the smartwatch accelerometer $\mathrm{z}$-axis \\
\hline 4 & $\begin{array}{l}\text { Number of the samplings that are greater than } 10.5 \\
\mathrm{~m} / \mathrm{s}^{2} \text { of the smartwatch accelerometer } \mathrm{x} \text {-axis }\end{array}$ \\
\hline 5 & $\begin{array}{l}\text { Number of the samplings that are less than } 9.1 \\
\mathrm{~m} / \mathrm{s}^{2} \text { of the smartwatch accelerometer } \mathrm{x} \text {-axis }\end{array}$ \\
\hline 6 & $\begin{array}{l}\text { Mean of the smartwatch accelerometer } \mathrm{x} \text {-axis after } \\
\text { ignoring the data that are laying between } 9.1 \text { and } \\
10.5 \mathrm{~m} / \mathrm{s}^{2}\end{array}$ \\
\hline 7 & Mean of the smartwatch gyroscope $\mathrm{x}$-axis \\
\hline 8 & Mean of the smartwatch gyroscope y-axis \\
\hline 9 & Mean of the smartwatch gyroscope $\mathrm{z}$-axis \\
\hline 10 & Mean of the smartwatch barometer sensor \\
\hline
\end{tabular}

\subsection{Classification}

As a result of the use of smartwatch fixed on human body ankle to obtain a distinguishable and separable raw data from the smartwatch sensors, the classifier of this work adopted a threshold-based mechanism to recognize human activity. Hence, the feature extraction stage separates essential features of each window according to the adopted classification scheme of each human activity. The extracted features of each window size (on the smartwatch) are sent to the smartphone, via Bluetooth, to be classified to the intended activity. the human activities that are recognized by this work are: Walking (slow, normal and fast), Jog, Run (slow, fast and run), Walking upstairs and downstairs, Elevator up and down, Sitting (completely or partially static), Sitting or Standing (completely or partially static) and Lying on his (left, right, stomach and back). Actually, due to the space limit of the paper, samples of the human activity classifications are discussed and analyzed in details in the following section.

\subsubsection{Walk, Jog and Run}

The smartwatch accelerometer and Gyroscope sensors are used to classify the walk, jog and run activities from other activities, such as walking upstairs/downstairs and static, in the following manner:

1-Figure 4 shows the raw data of the smartwatch accelerometer three axes signal variability for a walking activity for two window sizes. It is shown that as long as 
the accelerometer $\mathrm{x}$-axis is perpendicular to the earth and the user's foot is on the ground, hence the $x$-axis value of the accelerometer is approximately $9.8 \mathrm{~m} / \mathrm{s}^{2}$ $\left( \pm 0.7\right.$ greater or less than $\left.9.8 \mathrm{~m} / \mathrm{s}^{2}\right)$ which is first depicted in the figure from the period 1 to 10 . From period 11 to 50 the user has lifted his foot above the ground which is illustrated by the variation of the signal from approximately $4 \mathrm{~m} / \mathrm{s}^{2}$ to $20 \mathrm{~m} / \mathrm{s}^{2}$. In this period the $\mathrm{x}$ axis is no longer perpendicular to the earth, while the user is lifting her/his foot above the ground that produces different values for the $\mathrm{x}$-axis depending on the angle of the $x$-axis to the earth and the linear acceleration of the user's foot. In the consecutive periods from 51 to 66 and from 67 to 108 , nearly the same pattern mentioned above is repeated when the user's foot is on the ground and when the user is lifting her/his foot above the ground, respectively.

This work exploited this pattern to discriminate between the user's foot is on the ground or is lifted up. The other $y$-axis and z-axis signal variations do not yield a clear enough systematic pattern to be exploited for the mentioned activity recognition. Therefore, in order to achieve very high accuracy in discriminating between Walk, Jog and Run activities and other activities such as walking upstairs/downstairs, it is practically found that the best features that are unique and separable in the period are when the user is raising her/his foot above the ground.

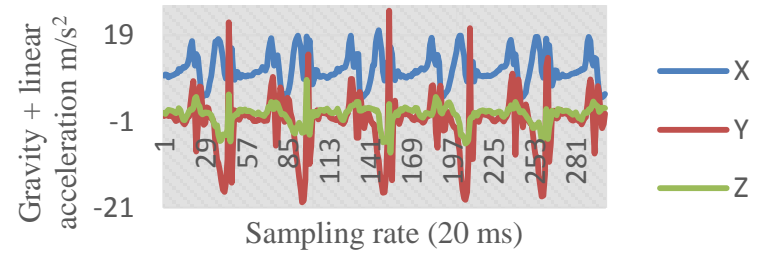

Figure 4: Raw data of the smartwatch accelerometer sensor for walk activity

2-Figures 5 and 6 show the raw data of the smartwatch accelerometer three axes signal variability of walking upstairs and downstairs activities, respectively, for two window sizes. Similar to the walking activity, the $\mathrm{x}$-axis is used to discriminate whether the user foot is on the ground or is lifted up. These similarities raise the problem of how to discriminate between the walk, jog, and run activities from one side and the walking upstairs/downstairs from another side. Actually, after conducting several tests, the following procedure is used to discriminate between the above-mentioned activities:

a. Ignoring all $\mathrm{x}$-axis readings of the smartwatch accelerometer sensor when their values laying between $9.1 \mathrm{~m} / \mathrm{s}^{2}$ and $10.5 \mathrm{~m} / \mathrm{s}^{2}( \pm 0.7$ greater and less than $9.8 \mathrm{~m} / \mathrm{s}^{2}$ ) because it is common in the other activities, such as walking upstairs/ downstairs and represents a period when the user is putting her/his foot on the ground. This period is different from user to another and it is also depending on the level of the user vitality.

b. After inspecting the raw data of the smartwatch accelerometer $x$-axis of the walk activity and walking upstairs/downstairs activities, it is found that the ratio of the number of samplings that are above than $10.5 \mathrm{~m} / \mathrm{s}^{2}$, in walk activity, to the number of samplings that are below than $9.1 \mathrm{~m} / \mathrm{s}^{2}$ is equal to or greater than 1.4 and the mean of the smartwatch accelerometer $\mathrm{x}$-axis is greater than 10.3 $\mathrm{m} / \mathrm{s}^{2}$. While in walking upstairs/downstairs activities, this ratio is less than 1.4 and the mean of the smartwatch accelerometer $\mathrm{x}$-axis is less than 10.3 $\mathrm{m} / \mathrm{s}^{2}$. Hence, after ignoring all data that are laying between $9.1 \mathrm{~m} / \mathrm{s}^{2}$ and $10.5 \mathrm{~m} / \mathrm{s}^{2}$, as mentioned in step (a) above, the system is able to distinguish and separate between the walk, jog and run activities from one side and the walking upstairs/downstairs activities from the other side.

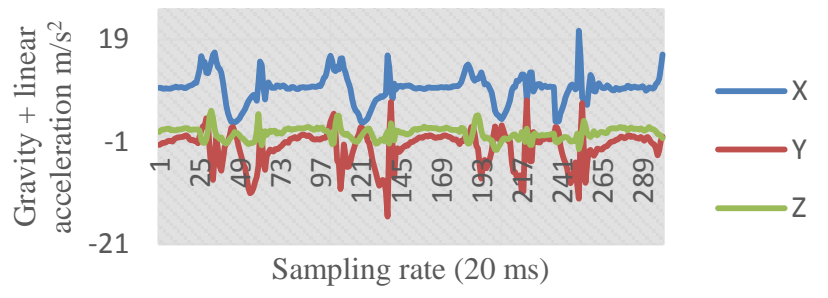

Figure 5: Raw data of the smartwatch accelerometer sensor for walking upstairs activity

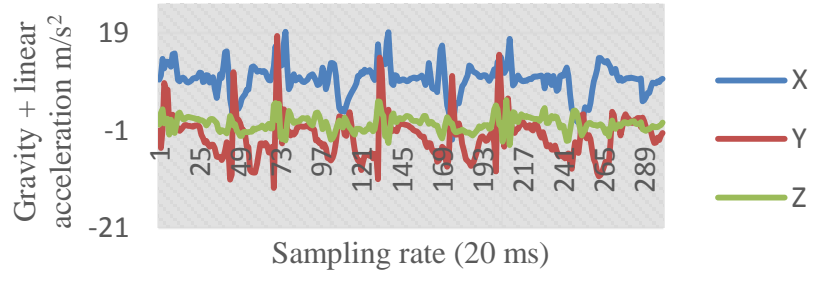

Figure 6: Raw data of the smartwatch accelerometer sensor for walking down stair activity

3-Figure 7 shows the statistics relationship procedure, mentioned in step 2 above, for separating the walk, jog and run activities from walking upstairs/downstairs activities. The $\mathrm{x}$-axis in the figure represents the ratio of a number of samplings that are above than $10.5 \mathrm{~m} / \mathrm{s}^{2}$ to the number of samplings that are below than $9.1 \mathrm{~m} / \mathrm{s}^{2}$. While the $y$-axis represents the mean value of the $x$-axis of the smartwatch accelerometer sensor, in $\mathrm{m} / \mathrm{s}^{2}$, excluding the number of samplings that are laying between 10.5 and $9.1 \mathrm{~m} / \mathrm{s}^{2}$. Each colored dot represents 
the ratio, mentioned above, of one window size for each activity walk, walking upstairs and downstairs. This test is conducted for 50 window sizes for a person doing the walk, walking upstairs and downstairs activities. It is noticed from the figure that the adopted procedure has discriminated the walking activity from walking downstairs/upstairs activities at threshold ratio of 1.4 and mean of $10.3 \mathrm{~m} / \mathrm{s}^{2}$.

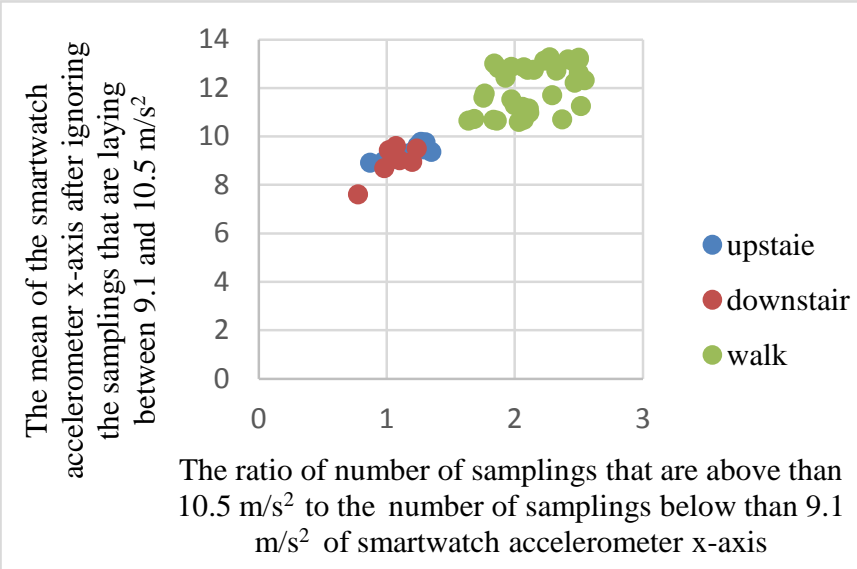

Figure 7: Separation between walk, and walking upstairs/downstairs activities

After having separated the Walk, Jog, and Run activities from other two activities walking upstairs and downstairs, the walking upstairs and downstairs activities include intervening points as shown in figure 7. The walking upstairs and downstairs activities are discriminated from each other using the smartwatch barometer sensor. After determining that the current activity falls in the domain of Walk, Jog, or Run. Now it is the time to separate between the walk (slow, normal, and fast), jog and run (slow, normal, fast) activities themselves which is accomplished by using the smartwatch gyroscope sensor. The gyroscope sensor is a motion sensor used to measures a device's rate of rotation in $\mathrm{rad} / \mathrm{s}$ around each of the three physical axes $(x, y$, and $z)$. The values of the Gyroscope three axes are positives and sometimes negatives depending on the direction of the rotation. The procedure that is used to discriminate between these activities is explained as follows:

1- Figures 8, 9 and 10 show the raw data of the smartwatch gyroscope three axes signal variability of the Walk, Jog, and Run activities, respectively, for two window sizes. The figures depict that the repetition of the Gyroscope three axes signal variations (for the same period) in the Walk activity is less than the Jog activity which in turn is less than the Run activity. The increased repetition of the signal variations for the Walk, Jog, and Run activities occurs because the movement speeds of these activities are different. The repetition of signal variations is increased whenever the speed of the user is increased. This phenomenon is observed clearly, for example, from the 3-axes signal variation shown in the figures mentioned above. It is also found that the sum of the mean values of the absolute three axes in walk activity is less than that of Jog activity and the latter is less than Run activity.

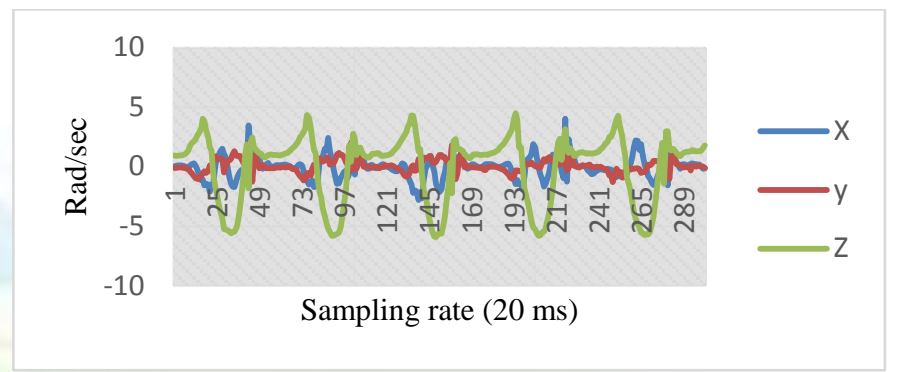

Figure 8: Raw data of the smartwatch Gyroscope sensor for walk activity

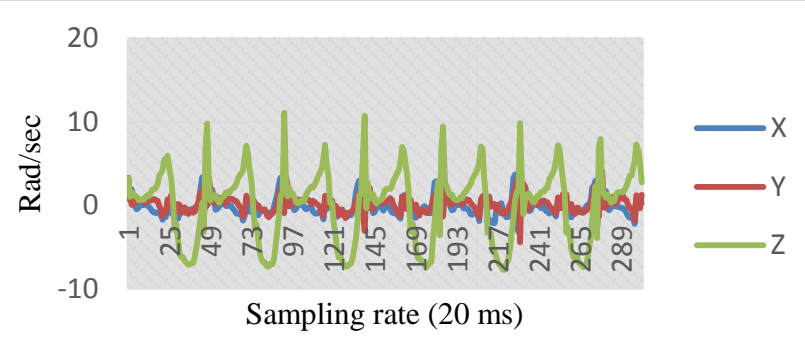

Figure 9: Raw data of the smartwatch Gyroscope sensor for Jog activity

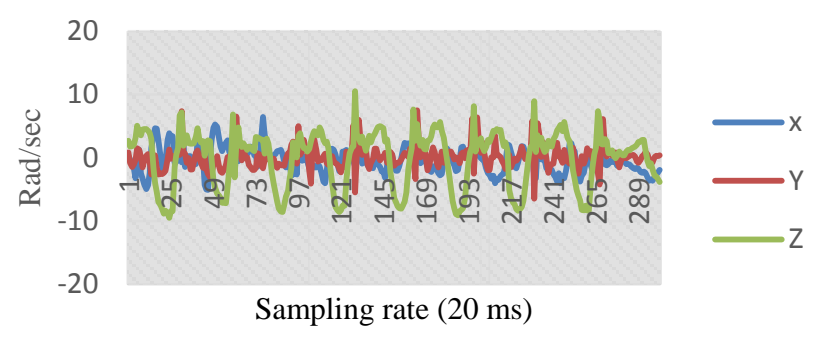

Figure 10: Raw data of the smartwatch Gyroscope sensor for Run activity

2- Hence, the procedure used to discriminate between the activities, mentioned in step 1 above, is by finding the sum of the mean values of each absolute Gyroscope three axes readings to provide motion level for each window size, and then applying a threshold-based value to recognize each activity as follows:

a. Walk slow: Threshold between [1.5 rad/s - 2.3 $\mathrm{rad} / \mathrm{s}$ ).

b. Walk normally: Threshold between $[2.3 \mathrm{rad} / \mathrm{s}-4$ $\mathrm{rad} / \mathrm{s}$ ).

c. Walk fast: Threshold between [4 $\mathrm{rad} / \mathrm{s}-5 \mathrm{rad} / \mathrm{s}$ ). 
d. Jog: Threshold between [5 rad/s $-6 \mathrm{rad} / \mathrm{s}$ ).

e. Run slow: Threshold between [6 rad/s - 6.5 $\mathrm{rad} / \mathrm{s}$ ).

f. Run normal: Threshold between $[6.5 \mathrm{rad} / \mathrm{s}-7.5$ $\mathrm{rad} / \mathrm{s}$ ).

g. Run fast: Threshold (greater than $7 \mathrm{rad} / \mathrm{s}$ ).

Figure 11 shows the variation of the sum of the mean values for the absolute Gyroscope three axes readings to provide motion level for 88 window sizes that illustrate the function of the Gyroscope in determining the above seven mentioned activities. The figure shows the motion level from the first window which represents that the user is completely static as long as the mean is zero, to window number 82 which represents the user is running fast.

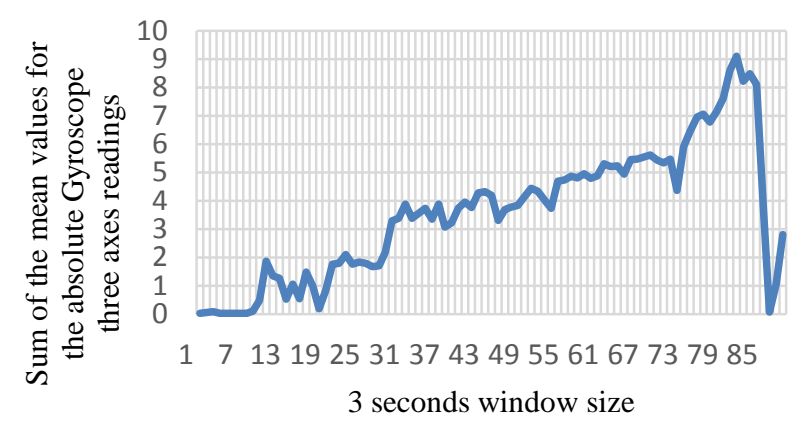

Figure 11: Sum of the mean values for the absolute Gyroscope three axes readings during the Walk, Jog, and Run activities
The rest of the human activities, presented in this work, are classified in a similar manner that a threshold based values are applied concerning the pattern which is produced from performing the intended activity.

\section{RESULTS AND PERFORMANCE}

Twenty human activities have been practically tested in this work. In order to reduce the amount of the results presented in the table, the test results are concentrated on the main human activities. For example, the subactivities of the walk (slow, normal and fast) are omitted and regarded as the only walk. In fact, these sub-activities are integrated into the system to represent the level of the motion which in turn is used to predict the level of the user vitality. Several experimental tests are conducted with 5-subjects (2male 2-female and 1-boy). The overall tests of each activity for whole users are represented in the confusion matrix shown in table 2. The type of the device used in this work test is Huawei smartwatch watch and HTC one smartphone. Also, this work has been tested using different types of Android platform smartphones like Galaxy 4 and Sony Xperia Z1. The results obtained from the three smartphones are fairly similar. 
Table 2 Overall confusion matrix for human activity arogrition

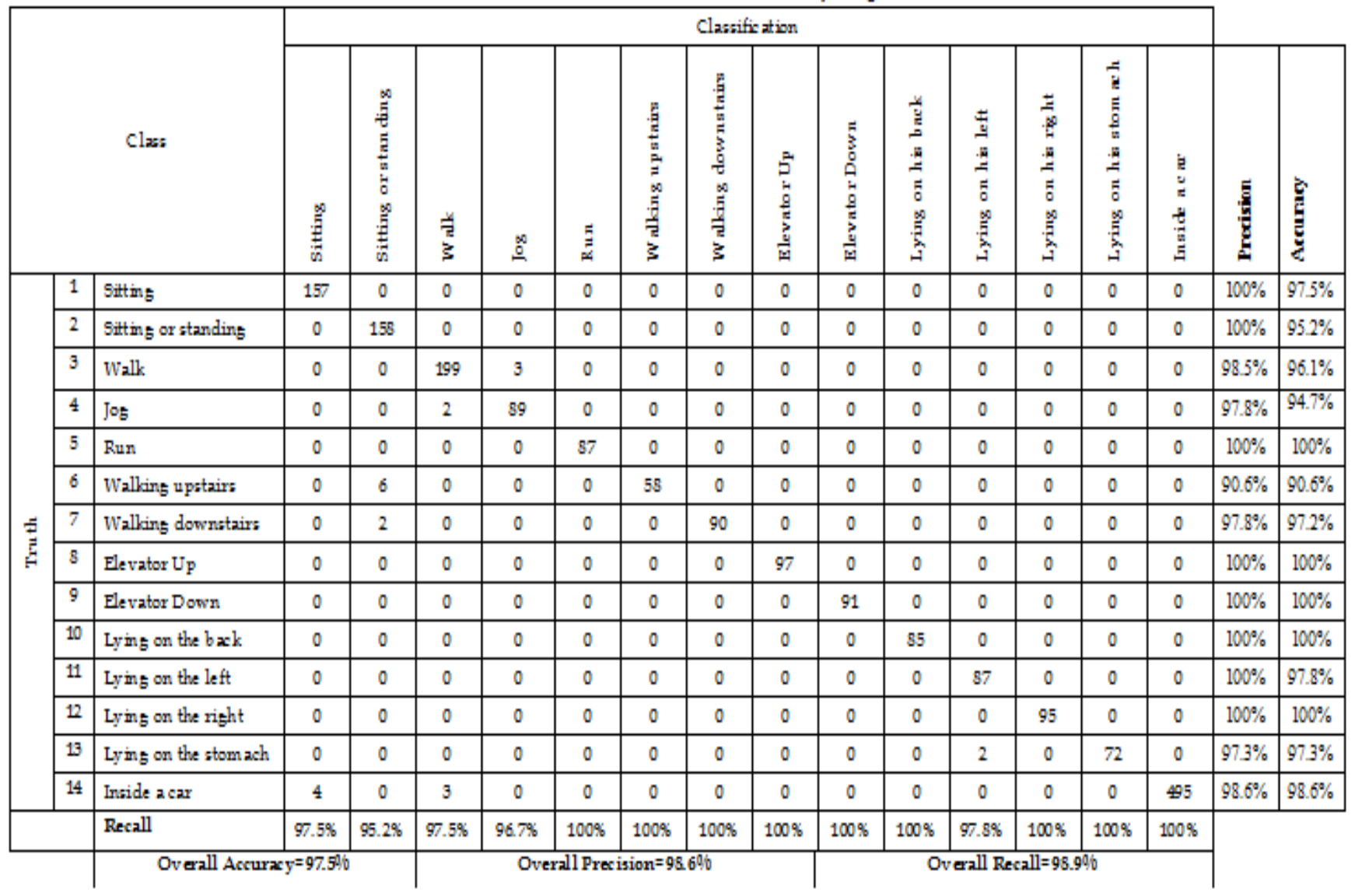

\section{CHALLENGES}

During the time of the system analysis and results inspection of the human activity recognition systems, it is found that some unpredictable phenomenon occurs during the time of recognition. Furthermore, there are other factors, such as window size and sampling rate, also affect the accuracy of the human recognition system. These phenomenon and factors are explained below:

1-The phenomenon that affects the classification of the human activities is the completion of the activity motion inside one window size. It happens that part of the activity motion occurs in a certain window and the another part occurs in the next window. In other words, the activity does not necessary start at the beginning of each window. For example, in a walking activity, a small part of the walk activity signal variation occurs in the current window, while the largest signal variation part occurs in the next window in which the user starts the walking upstairs activity. Hence, because of the effect of the walk signal variation, the latter window could be misclassified as a walking activity. Hence, sometimes it is very difficult to achieve 100\% classification accuracy even if distinct and separable features obtained from the sensors. In fact, it is impossible to synchronize the start and end of each activity time with the start and end of each window size. Also, when the user transition time between different activities is increased, then the probability of classification accuracy is decreased.

2-Choosing adequate time for the window size is very crucial subject because there are many pros and cons when increasing or decreasing the time of the window size:

a-When the activities are changing in a short period of time (less than one window size) then the ability to reorganize such activities is decreased when increasing the window size, and vice versa.

b-The accuracy of recognizing human activities will increase when increasing the time of the window size because sufficient and clear features will be available, and vice versa.

c- The amount of the database storage will decrease when increasing the time of the window size, and vice versa. 
d-The computation power of the system will decrease when the time of the window size is increased and vice versa.

e-Increasing the time of the window size is not suitable for real-time monitoring.

3-The other factor that affects the accuracy of the recognition system is the sampling rate which has the following pros and cons:

a-Increasing the sampling rate will increase the accuracy of the human recognition system, and vice versa.

b-Increasing the sampling rate will increase the computation power of the system, and vice versa.

\section{SMARTWATCH ROLE}

Actually, most of the works done in the field, of human activity recognition, adopted the approaches of using smartphone alone or smartphone together with an external sensor. These approaches associated with difficulties and obstacles that prevent them from achieving flexible and highly accurate systems. These difficulties are analyzed and justified as follows:

1-Due to the change in smartphone position and orientation, the human activity recognition system does not work properly when the smartphone is freely held by the user's hand or in a bag as most women do.

2-In order to obtain, to a certain degree, distinct signal variation from the smartphone sensors, all the related works have imposed the restriction of carrying the smartphone in a fixed position on the human body, such as inside trousers pocket or on the chest. This restriction has the following drawbacks and shortcomings:

a- Sometimes the user wants to use the smartphone for the purpose of calling, serving the internet, social media, social networks, knowing the weather, camera etc. Moving the smartphone from its fixed position impose a wrong interpretation of the smartphone sensor coordinates due to the change in smartphone position and orientation.

b- The smartphone in the human pocket is not settled and to somewhat changing its orientation due to the difference in the type of the clothes. Also, most of the pockets are not tight and small enough to prevent the smartphone from changing its position and orientation during the user movement.
3-Due to the analyses mentioned in step 2 above, and because the features obtained (from solely using the smartphone sensors) are not generalized and separable, an artificial intelligent classifier is needed to predict and discriminate each activity features. The burdens of using artificial intelligent classifier are:

c- The recognition system must be trained for each user or many users.

$\mathrm{d}$-The training data and the classifier must be loaded into where the processing will take place, smartphone, networked computer.

e-The artificial intelligent classifier does not guarantee accurate recognition results due to the different situation of the smartphone when it is fixed on the human body.

f- Also, extra processing time is required by the artificial intelligent classifiers to accomplish their duty.

e-The signal variation of the accelerometer and gyroscope, for the user performing walking upstairs and downstairs activities, are varying relative to the type and structure of the stairs. Hence, in the systems that depend on the training data set, the accuracy of walking upstairs and downstairs recognition depends on the type of the satires used in the training data set. In other words, the system could be prone to error when different types of stairs are conducted by the user.

4-The activity recognition system does not work properly and sticks to only one activity, when inadvertently people leave their smartphones, most of the time, on a table when they are working or lying.

The main contribution of this work is the incorporation of the android smartwatch as a part of the system components. From the practical test, it is found that the smartwatch, fixed on the human ankle, is the best external sensor that can be used in conjunction with a smartphone for better accurate human activity recognition results. Justifications and the rational reasons behind the use of the smartwatch as part of the proposed system components, compared to the smartphone alone or smartphone with external dedicated sensor, are discussed as follows:

1-Smartwatch is equipped with some sensors that are not available on most smartphones, such sensors like barometer sensor, which is used in this work to discriminate between walking upstairs and 
downstairs, and heart rate sensor that can be used for heart rate checking.

Due to the smartwatch specifications like light weight, small size, water resistance and wireless communication, hence it can be placed and fixed in a position that generates generalized and separable features from its sensors. Figure 12 shows the smartwatch fixed on human ankle compared to the smartphone fixed on the human trousers pocket in a walking activity. It is realized and observed from the figure that the range of displacement and orientation performed by the smartwatch are much wider and bigger than in a smartphone. The range of the smartwatch displacement and orientation makes it generates notable and distinguishable signal variations by its accelerometer and gyroscope sensor coordinates.

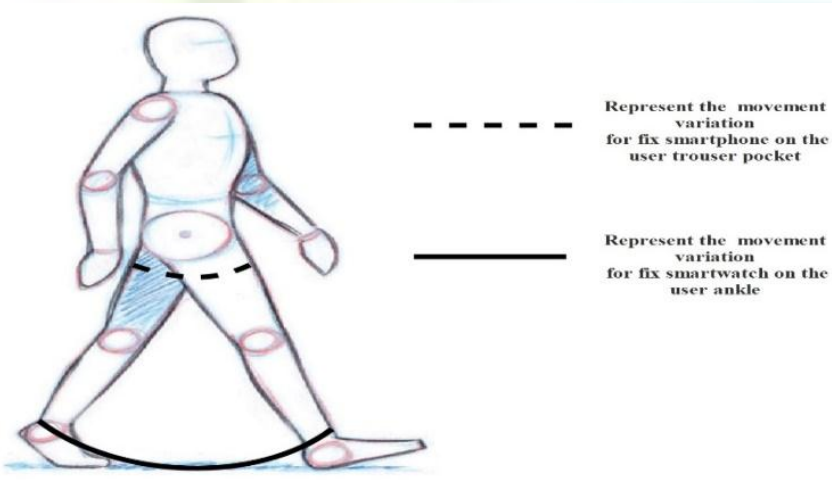

Figure 12: Represent the user variation for fixed smartwatch and fixed smartphone

2-Unlike fixed smartphone on the human body, the proposed smartwatch system is continuously working even when the smartphone is in use for other purposes such calling, internet serving, or even when the smartphone placed on a table near to the user.

3-The use of the external sensor in conjunction with the smartphone, like Zephyr Bio Harness, is too expensive and is not Android platform compatible; also it does not contain all the sensors of the smartwatch.

4-Fixing the smartphone position and orientation on the human body is not feasible due to the size of the smartphone in comparison with smartwatch

5 -Due to the processing capability of the smartwatch, it minimizes, to a large degree, the computation power load on the smartphone by performing most of the feature extraction process on the smartwatch rather than the smartphone. The processing capability of the smartwatch is not available in the external wearable sensors.
6-The smartwatch minimizes the amount of data (network load) transferred from the smartwatch to the smartphone. Actually, only the extracted features of each window size (of 3 seconds) are sent to the smartphone. Hence, the extracted features are sent once every 3 seconds rather than sending the smartwatch raw data every $20 \mathrm{~ms}$.

\section{CONCLUSION}

During the study and development of human activity recognition system, several points observed and noticed:

1- It is realized and concluded that for such system to work properly, then it is necessary and compulsory obtaining clear distinguishable signal variations from the smartphone sensors such that 3-axes accelerometer and gyroscope. In response to that, all the like systems have imposed the restriction of fixing the smartphone on the human body.

2- Fixing the smartphone on the human body is not practical and is not the ultimate solution for such systems to work properly which is also prone to a partial movement that could affect the signal variation of the smartphone sensors.

3- Furthermore, the variation in the pattern movement of each activity from user to user due to the change in the user gender and age, also within the same user due to the change in the user vitality and mood makes it hard and difficult to achieve subject independent and accurate real-time human activity recognition system.

4- Consequently, integrating the smartwatch (steady fixed on the human angle) with a smartphone is due to the main reason of obtaining generalized and separable features for a wide number of activities with very high accuracy.

5- For future work, to overcome the misclassification that arises from using fixed window size, a variable window size can be studied and incorporated in such system that prevents the intervening of two activities in one window size.

\section{REFERENCES}

[1] S. Adibi, "Introduction," in Mobile Health A Technology Road Map, Springer International Publishing Switzerland, 2015, p. 1.

[2] R. Amin, C. Chien-An and L. Yan, "Human Activity Recognition using Smartphone," CSCE666, 2011.

[3] K. Mustafa, I. Ozlem Durmaz and E. Cem, "Online Human Activity Recognition on Smart Phones," in 2nd International Workshop on Mobile Sensing, Beijing, China, 2012.

[4] H. Yi and L. Ye, "Physical Activity Recognition Utilizing the Built-In Kinematic Sensors of a Smartphone," International Journal of 
Hamid M. Ali et al. ," Human Activity Recognition Using Smartphone and Smartwatch", International Journal of Computer Engineering In Research Trends, 3(10):568-576,October-2016. DoI:10.22362/ijcert/2016/v3/i10/48906

Distributed Sensor Networks, vol. 9, no. 1550-1477, p. 10, 2013.

[5] A. Davide, G. Alessandro, O. Luca, P. Xavier and L. R.-O. Jorge, "A Public Domain Dataset for Human Activity Recognition Using Smartphones," in European Symposium on Artificial Neural Networks, Computational Intelligence and Machine Learning, Bruges Belgium, ESANN, 2013, pp. 437-442.

[6] Y. Bingchuan, H. John and E. Yalda, "Smartphone-based Activity Recognition Using Hybrid Classifier-Utilizing Cloud Infrastructure for Data Analysis," in Proceedings of the 4th International Conference on Pervasive and Embedded Computing and Communication Systems, Lisbon, Portugal, 2014.

[7] T. H. Cesar and A. L. Andres, "Accelerometer-Based Human Activity Recognition in Smartphones for Healthcare Services," in Mobile Health A Technology Road Map, Springer International Publishing Switzerland, 2015, p. 147.

[8] D. Natarajasivan and M. Govindarajan, "Filter Based Sensor Fusion for Activity Recognition using Smartphone," International Journal of Computer Science and Telecommunications, vol. 7, no. 5, pp. 2631, 2016. 